\title{
Large-Grained Polycrystalline (111) Ge Films on Insulators by Thickness-Controlled Al-Induced Crystallization
}

\author{
K. Nakazawa, ${ }^{a}$ K. Toko, ${ }^{a, z}$ N. Saitoh, ${ }^{\text {b }}$ N. Yoshizawa, ${ }^{b}$ N. Usami, ${ }^{c}$ and T. Suemasu ${ }^{a}$ \\ ${ }^{a}$ Institute of Applied Physics, University of Tsukuba, Tsukuba, Ibaraki 305-8573, Japan \\ ${ }^{b}$ Electron Microscope Facility, IBEC Innovation Platform, AIST, Tsukuba 305-8569, Japan \\ ${ }^{c}$ Institute for Materials Research, Tohoku University, Sendai, Miyagi 980-8577, Japan
}

\begin{abstract}
Low-temperature $\left(350^{\circ} \mathrm{C}\right)$ crystallization of amorphous $\mathrm{Ge}$ films on $\mathrm{SiO}_{2}$ was investigated using Al-induced layer exchange (ALILE) process. Thicknesses of Ge and catalytic Al layers were varied in the range of 30-300 nm, which strongly influenced the ALILE growth morphology. Based on the study, the Ge thickness was adjusted to $40 \mathrm{~nm}$ while the Al thickness was adjusted 50 nm. This sample satisfied both of the surface coverage of polycrystalline-Ge and the annihilation of randomly oriented Ge regions. Moreover, the enhancement of the heterogeneous Ge nucleation improved the (111) orientation and the grain size. As a result, the area fraction of the (111)-orientation reached as high as $97 \%$ and the average grain size as large as $70-\mu \mathrm{m}$ diameters. This (111)-oriented Ge layer with large-grains promises to be the high-quality epitaxial template for various functional materials to achieve next-generation devices.

(C) 2013 The Electrochemical Society. [DOI: 10.1149/2.007311jss] All rights reserved.
\end{abstract}

Manuscript submitted May 22, 2013; revised manuscript received August 1, 2013. Published August $13,2013$.

Germanium-on-insulators are essential for high-speed thin-film transistors, highly-efficient solar cells, and three-dimensional on-chip optical interconnects. ${ }^{1-3}$ Some applications require low-temperature processes to avoid softening glass substrates $\left(<500^{\circ} \mathrm{C}\right)$ or breaking underlying integrated circuits $\left(<400^{\circ} \mathrm{C}\right)$. In particular, (111)-oriented Ge film is favorable for developments of high-speed Ge transistors, nanowires of Ge or III-V compound semiconductors, and spintronics materials. ${ }^{4-8}$ In line with this, metal-induced crystallization (MIC) of amorphous $\mathrm{Ge}$ (a-Ge) films on insulators has been studied for over a decade. ${ }^{9-13}$ In the MIC, catalytic metals ( $\mathrm{Ni}, \mathrm{Au}, \mathrm{Cu}$, etc.) enable the low-temperature crystallization of a-Ge in the temperature range $150-400^{\circ} \mathrm{C},{ }^{9-13}$ which are significantly lower than the crystallization temperatures of a-Ge without the metals., ${ }^{2,14,15}$ In particular, a nanowire-like single-crystal $\mathrm{Ge}$ is achieved by metal-induced lateral crystallization (MILC), which is promising for high-speed Ge transistors. ${ }^{10}$ However, for large-area devices such as solar cells, these growth techniques are not applicable due to the small grains or the narrow growth regions. Therefore, the enlargement of the grain size is required because the grain boundaries deteriorate the device performances. ${ }^{15,16}$

Aluminum-induced layer exchange (ALILE) is one of the MIC techniques developed for amorphous-Si (a-Si) films on insulators. ${ }^{17-26}$ This ALILE technique enables us to form large-grain (diameters: $10-100 \mu \mathrm{m})$ polycrystalline Si films at relativity low temperatures $\left(420-550^{\circ} \mathrm{C}\right)$ through the exchange between the $\mathrm{Al}$ and a-Si layers during annealing. ${ }^{17-24}$ Moreover, the orientation of the grown Si film can be controlled to either (100) or (111) plane by modulating the annealing temperature ${ }^{19,20}$ and the thickness of the interlayer between $\mathrm{Si}$ and Al layers. ${ }^{21,23}$ The chemical vapor deposition (CVD) of $\mathrm{Si}$ layers onto the ALILE-Si films is a promising approach to fabricate large-grained Si light-absorption layers on glass substrates. ${ }^{25,26}$

Some researchers applied this ALILE technique to a-Ge films on insulators and succeeded to reduce the crystallization temperature. ${ }^{27-31}$ However, the layer exchange was not achieved due to the absence of the interlayer between $\mathrm{Ge}$ and $\mathrm{Al}$ layers. This resulted in small-gained $\mathrm{Ge}-\mathrm{Al}$ mixed layers with random orientations. $\mathrm{Hu}$ et al. and Kurosawa et al. recently achieved the layer exchange by fabricating the proper interlayer between $\mathrm{Ge}$ and $\mathrm{Al}$ layers. ${ }^{32,33}$ Moreover, we have significantly improved the crystal quality of the ALILE-Ge films by lowering the annealing temperature below $350^{\circ} \mathrm{C} .{ }^{34,35}$ Meanwhile, some papers on the ALILE imply the important role of the film thickness in the growth morphorogy. ${ }^{17,21,33}$ For Si, Nast et al. reported that the thickness ratio of $\mathrm{Al}$ and $\mathrm{Si}$ should be 1:1 for a good layer exchange. ${ }^{17}$ With the thickness ratio of $1: 1$, Kurosawa et al. found that the thinner Si and Ge layers yield the higher (111) orientation fraction

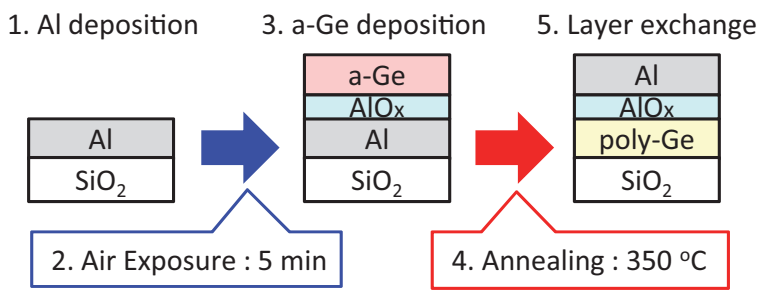

Figure 1. Schematic structure of the sample preparation procedure.

in the ALILE-Si and Ge layers. ${ }^{21,33}$ However, for the ALILE-Ge, the growth condition, that is the $\mathrm{AlO}_{\mathrm{x}}$ formation process and the annealing temperature, were not optimized, which resulted in relatively low (111) orientation fraction (70\%) and small grains (approx. $5 \mu \mathrm{m}){ }^{33}$ In this paper, we have investigated in detail the effects of the $\mathrm{Ge} / \mathrm{Al}$ thickness on the ALILE-Ge under the recently-established growth conditions: the $\mathrm{AlO}_{\mathrm{x}}$ interlayer and the low-temperature annealing $\left(350^{\circ} \mathrm{C}\right)$. We demonstrate appropriate $\mathrm{Al}$ and $\mathrm{Ge}$ thicknesses from the view point of the grain size and the (111) orientation fraction.

\section{Experimental}

The sample preparation procedure is schematically shown in Fig. 1. Al films were first prepared onto quartz glass $\left(\mathrm{SiO}_{2}\right)$ substrates. Subsequently, the samples were exposed to air for $5 \mathrm{~min}$ to form native $\mathrm{AlO}_{\mathrm{x}}$ layers as diffusion control layers. After that, a-Ge films were deposited. The thicknesses of $\mathrm{Al}$ and a-Ge layers were varied from $30 \mathrm{~nm}$ to $300 \mathrm{~nm}$. The thickness combinations of $\mathrm{Al}$ and Ge layers are summarized in Table I. Here, these values have a margin of error of plus or minus 5\%. All the depositions were carried out at room temperature using a radio-frequency magnetron sputtering method. Finally, those samples were annealed at $350^{\circ} \mathrm{C}$ for $30 \mathrm{~h}$ in a $\mathrm{N}_{2}$ ambient.

A $\theta-2 \theta$ X-ray diffraction (XRD) measurement was carried out to evaluate the crystal states of the grown Ge layers. Surface morphologies and crystal orientations were respectively evaluated using scanning electron microscopy (SEM) and electron backscattered

Table I. Thicknesses of $\mathrm{Ge}$ and $\mathrm{Al}$ layers prepared on $\mathrm{SiO}_{2}$ substrates.

\begin{tabular}{ccccccccc} 
Sample & A & B & C & D & E & F & G & H \\
\hline Ge thickness (nm) & 40 & 100 & 40 & 30 & 50 & 100 & 300 & 40 \\
Al thickness (nm) & 40 & 40 & 100 & 30 & 50 & 100 & 300 & 50
\end{tabular}


diffraction (EBSD) measurements. Prior to SEM and EBSD, the aluminum and oxide layers on the Ge layers were etched away by diluted HF solutions (HF: $1.5 \%$ ) for $1 \mathrm{~min}$.

\section{Results and Discussion}

Fig. 2 shows the XRD spectra of sample A (40-nm-thick Ge, 40nm-thick Al) before and after annealing. Before annealing, no peak is present in the spectra. In contrast, a sharp peak appears at around $27^{\circ}$ after annealing corresponding to the Ge (111) plane. Any other peak is not observed in the measured $2 \theta$ range $\left(20-80^{\circ}\right)$. These results indicate that the a-Ge layer was crystallized during annealing, and the grown Ge layer is preferentially oriented to the (111) plane. The precise (111)-orientation fraction will be estimated later by means of EBSD measurements. The inserted photograph shows that the colors of the sample surfaces differed after the annealing. This suggests that the top layer changed from $\mathrm{Ge}$ to $\mathrm{Al}$ during annealing. This is a typical feature of the successful ALILE process. ${ }^{18}$

We investigated the effect of the $\mathrm{Ge}-\mathrm{Al}$ thickness ratio on the growth morphology of the ALILE. Three samples were prepared: sample A with 40-nm-thick Ge and 40-nm-thick Al, sample B with 100-nm-thick Ge and 40-nm-thick Al, and sample C with 40-nm-thick Ge and 100-nm-thick Al, as summarized in Table I. SEM and EBSD images of the sample surfaces are exhibited in Fig. 3. These EBSD images show the crystal orientations along the normal direction (ND) to the sample surface. Al layers were removed before these measurements. By comparing Figs. $3 a$ and $3 b$, we found that some randomly oriented Ge regions are present inside the (111)-oriented Ge layer. Meanwhile, for sample B, the randomly oriented region covers almost the entire region of the sample surface, as shown in Figs. 3c and $3 \mathrm{~d}$. For sample $\mathrm{C}$, the insufficient Ge coverage is observed in Fig. 3e. However, the Ge layers have very little randomly oriented region, and thus the (111)-orientation is quite dominant as shown in Fig. 3f. Now, we summarize the Ge-Al thickness-ratio dependence of the growth morphology of the ALILE. The higher the Ge proportion, the larger the randomly oriented regions. In contrast, the higher the Al proportion, the smaller the randomly oriented regions. This suppression of the randomly oriented regions results in the dominant (111)-orientation, however, the surface coverage of Ge becomes insufficient. These results suggest that the further optimization of the $\mathrm{Ge}-\mathrm{Al}$ thickness ratio is required to achieve the fine growth morphology.

Fig. 4a shows a typical bright-field TEM cross-section of sample A before the $\mathrm{Al}$ etching, which was observed in $\mathrm{Ge}[-1-10]$ zone axis. Fig. $4 \mathrm{~b}$ and $4 \mathrm{c}$ show selected area electron diffraction (SAED) patterns observed at the areas shown in Fig. 4a. From these results, we can see that the left area in Fig. 4 a consists of a (111)-oriented bottom-Ge layer and a top-Al layer, while the right area consists of a (111)-oriented bottom-Ge layer and a top-Ge layer. This top-Ge layer probably corresponds to the randomly oriented Ge regions shown

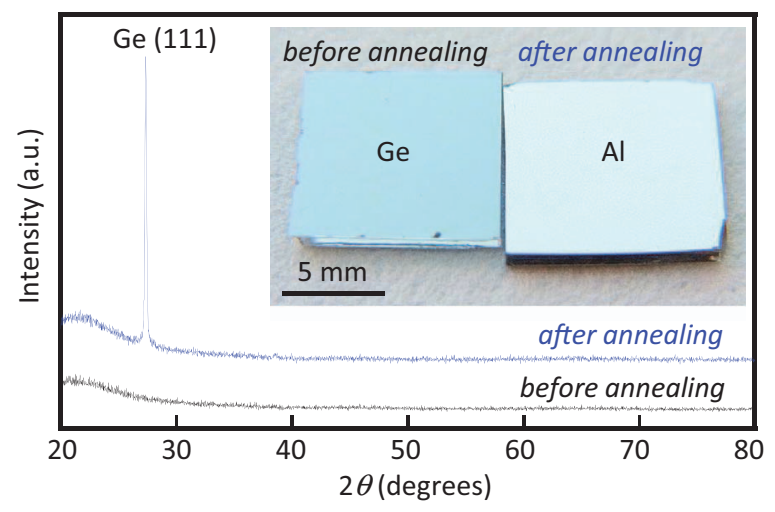

Figure 2. XRD patterns of the sample before and after annealing. Inserted photograph shows the sample surface before and after annealing.

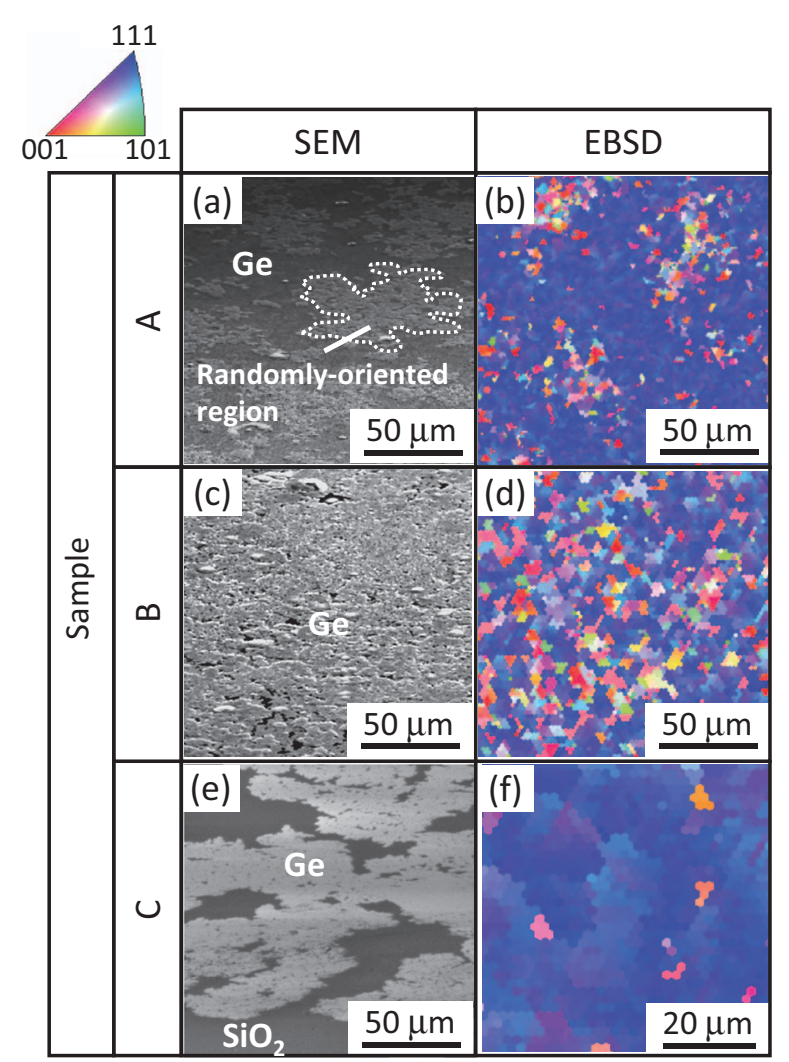

Figure 3. Surface morphologies and crystal orientations for samples A, B, and $\mathrm{C}$, prepared with various Ge-Al thickness ratios. (a)-(f) SEM and EBSD ND images of the grown Ge layers. Al layers were removed before these measurements. Inserted color-key corresponds to crystal orientations.
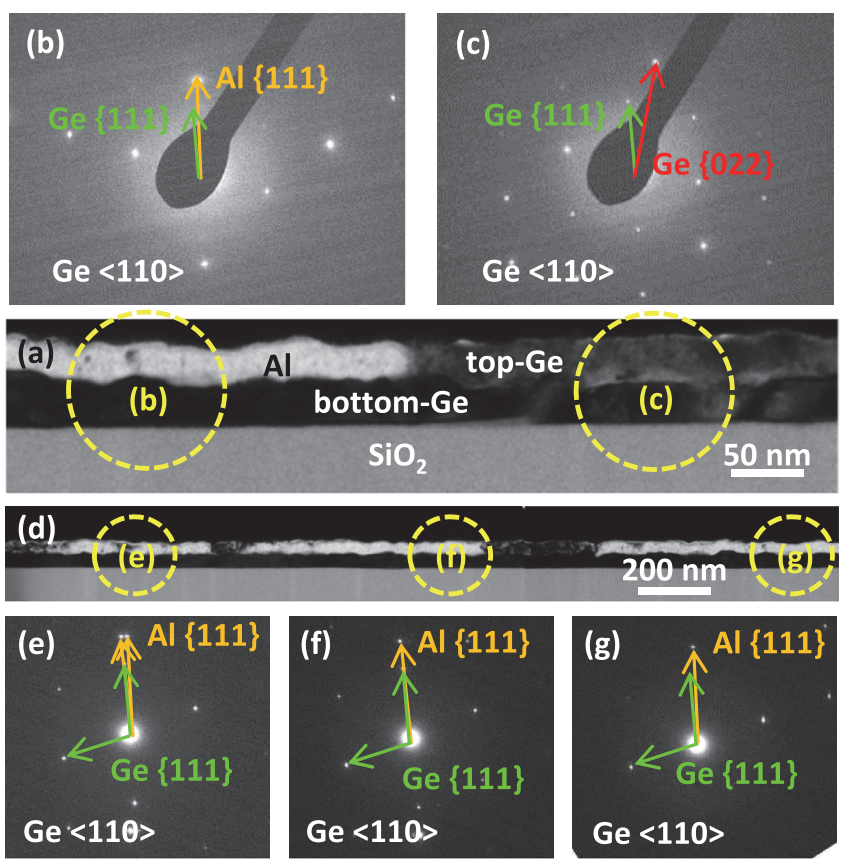

Figure 4. Characterization of the cross-section structure of sample A. (a) Bright-field TEM image. (b), (c) SAED patterns obtained at the areas shown in (a). (d) Low-magnified bright-field TEM image. (e), (f), (g) SAED patterns obtained at the areas shown in (d). 


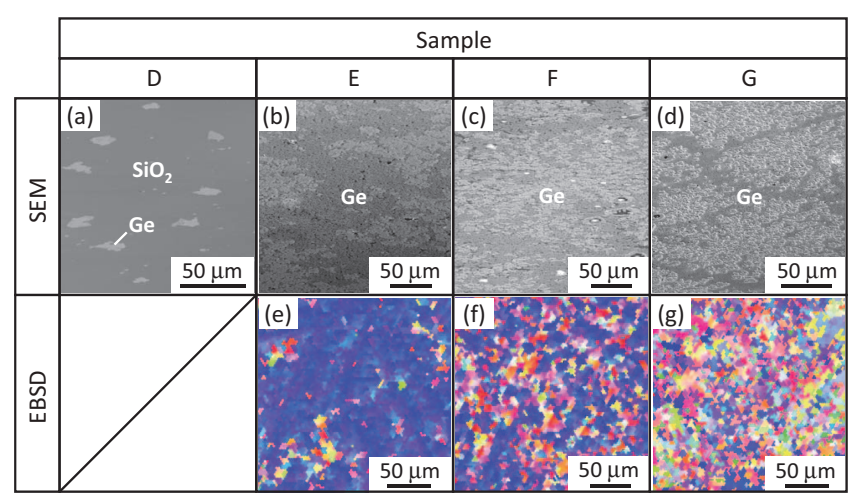

Figure 5. Surface morphologies and crystal orientations for samples D, E, F, and $\mathrm{G}$, prepared with the Ge-Al thickness ratio of 1:1. (a)-(g) EBSD ND and SEM images of the grown Ge layers. Inserted color-key corresponds to crystal orientations.

in Fig. 3. Fig. 4d shows a low-magnified TEM image of the Ge/Al stacked region. We confirmed the absence of random grain boundaries in the bottom-Ge layer. In addition, SAED patterns shown in Figs. $4 \mathrm{e}-4 \mathrm{~g}$ indicate that the bottom-Ge layer has exactly the same orientation, whereas the Al layer consists of some different grains with (111) orientation. Here, a big bright dot at the center of SAED pattern means a direct electron beam which penetrates a TEM specimen without any interaction. On the other hand, only diffracted electron beams which satisfy Bragg's law are indicated on SAED as diffraction spots. For this reasons, the intensity of the direct electron beam is much higher than diffraction spots. Consequently, these results prove the single-crystal Ge at least in this observed area (approx. $2.5-\mu \mathrm{m}$ width). Because the top and bottom Ge layers have no epitaxial relationship, growth process of those layers should be totally different. The growth mechanism is discussed later.

Next, the effects of absolute thickness values of Ge/Al layers, with 1:1 thickness ratio, were investigated by preparing samples D-G. The $\mathrm{Ge} / \mathrm{Al}$ layer thicknesses of these samples are summarized in Table I. SEM and EBSD images of the sample surfaces are exhibited in Fig. 5. These EBSD images show the orientations along ND to the sample surface. Al layers were removed before these measurements. For sample D, with 30-nm-thick Al and Ge layers, the nucleation and lateral growth of $\mathrm{Ge}$ stopped during annealing. As a result, grown Ge areas $(\sim 15-\mu \mathrm{m}$ diameters $)$ are distributed on the $\mathrm{SiO}_{2}$ substrate as shown in Fig. 5a. The EBSD measurement was difficult for this sample because of charge-up of the electrons on the insulating $\mathrm{SiO}_{2}$ substrate. For samples E (50-nm thickness), F (100-nm thickness), and $\mathrm{G}$ (300-nm thickness), grown Ge layers cover the $\mathrm{SiO}_{2}$ substrates as shown in Figs. 5b-5d. Randomly oriented Ge regions appear on the surfaces like in sample A. The EBSD images shown in Figs. 5e-5g indicate that the crystal orientation becomes random with increasing the Ge/Al thickness. This tendency agrees with the previous studies on the ALILE of a-Ge. ${ }^{33}$

For samples A, E, F, and G, with the Ge-Al thickness-ratio of 1:1, we evaluated the (111)-oriented area fraction and the grain size. Here, a grain was defined as a region surrounded by random grain boundaries, which region should be formed as a result of lateral growth from a single nucleus. The expected cross-sectional structure of these samples is schematically shown in Fig. 6a. Top-Ge layers, namely, randomly oriented Ge regions, are stacked on a bottom-Ge layer. Figs. 6b and $6 \mathrm{c}$ typically show low-magnified EBSD images for sample A along the normal direction (ND) and the transverse direction (TD) to the substrate, respectively. In Fig. 6c, random-grain boundaries corresponding to the bottom-Ge layer are indicated by the black solid lines. These lines were drawn based on EBSD analysis in order to evaluate the grain size of the bottom-Ge layer. EBSD analyzes derived the (111)-oriented area fractions from the ND orientation maps shown in Fig. 3b and Figs. 5e-5g. The result is summarized in Fig. 6d as a function of the Ge/Al thickness. Here, the (111)-oriented grains (a)
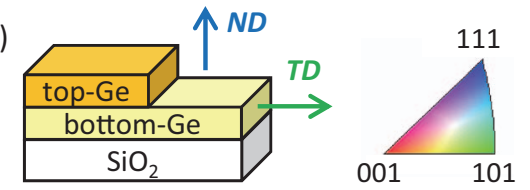

(b) ND
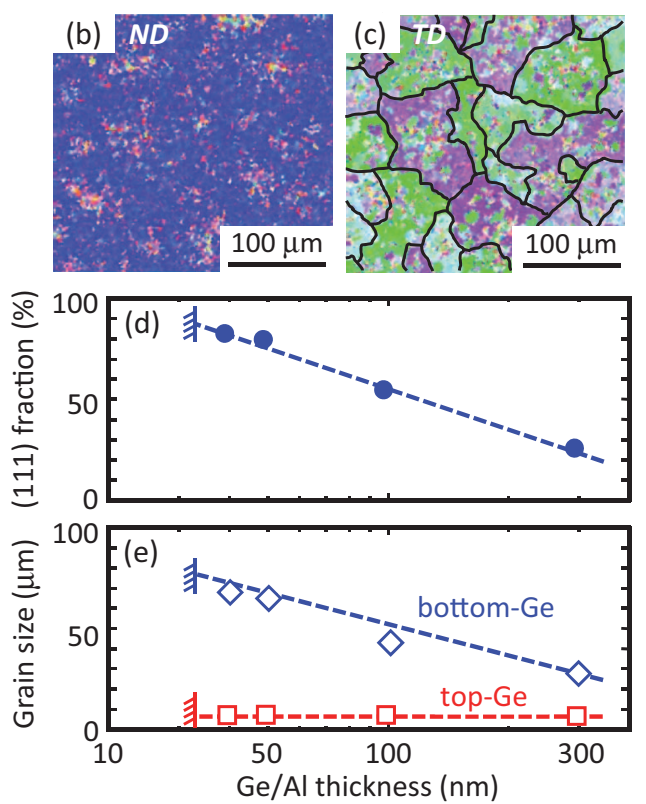

Figure 6. (a) Schematic image of the expected structure of ALILE-Ge. Lowmagnified EBSD images of sample A obtained along (b) ND and (c) TD. (d) Ge/Al thickness dependences of (111)-orientation fractions. (e) Ge/Al thickness dependences of grain sizes. The Ge-Al thickness ratios of these samples are 1:1. Inserted color-key corresponds to crystal orientations.

were defined as grains whose misorientation from the exact (111) plane was within $10^{\circ}$. Fig. $6 \mathrm{~d}$ clearly indicates that the (111) fraction increases with decreasing the Ge/Al thickness. The maximum (111) fraction reaches over $80 \%$ at $40-\mathrm{nm} \mathrm{Ge} / \mathrm{Al}$ thickness. Remember that the ALILE process cannot be completed when the Ge/Al thickness is below $30 \mathrm{~nm}$ as shown in Fig. 5a.

The grain sizes of the top and bottom Ge layers were derived from the EBSD images along the TD to the sample surfaces. The grain sizes of the bottom Ge layers were measured from the black lines in Fig. 6c. For the top Ge layers, grain sizes were measured from high-magnified EBSD images along TD. The average grain sizes (grain diameters) for the top and bottom Ge layers are summarized in Fig. 6 e as a function of the Ge/Al thickness. For the top-Ge layers, the grain size is small $(\sim \mu \mathrm{m})$, and remains almost unchanged regardless of the Ge/Al thickness. Meanwhile, for the bottom-Ge layers, the grain size clearly increases with decreasing the Ge/Al thickness. The maximum average grain size reaches $68 \mu \mathrm{m}$ in sample A, 40-nm $\mathrm{Ge} / \mathrm{Al}$ thickness. These thickness dependences of the orientation and grain size are summarized as follows: The thinner the Ge/Al thickness, the larger the grain size and the higher the (111)-orientation fraction. The lower limit of the thickness is between $30 \mathrm{~nm}$ and $40 \mathrm{~nm}$.

We discuss the growth mechanism on the thickness dependent $\mathrm{Ge}$ ALILE, based on the well-investigated Si-ALILE mechanism. ${ }^{19,22}$ Schematic growth mechanisms of samples with thin and thick $\mathrm{Ge} / \mathrm{Al}$ layer thicknesses are respectively illustrated in Figs. $7 \mathrm{a}$ and $7 \mathrm{~b}$. When $\mathrm{Ge}$ and $\mathrm{Al}$ layers are thin, diffusing $\mathrm{Ge}$ atoms reach the $\mathrm{SiO}_{2}$ surface before the $\mathrm{Ge}$ concentration in $\mathrm{Al}$ reaches the solubility limit. Hence, the Ge nucleation heterogeneously occurs at the $\mathrm{SiO}_{2}$ surface as schematically shown in Fig. 7a. These Ge nuclei are oriented to the (111) plane because the Ge (111) plane has the minimum surface energy on $\mathrm{SiO}_{2} \cdot{ }^{36}$ In contrast, when Ge and $\mathrm{Al}$ layers are thick, the Al layer becomes supersaturated with $\mathrm{Ge}$ before diffusing $\mathrm{Ge}$ atoms reach the $\mathrm{SiO}_{2}$ surface. Hence the homogeneous Ge nucleation occurs 
(a)
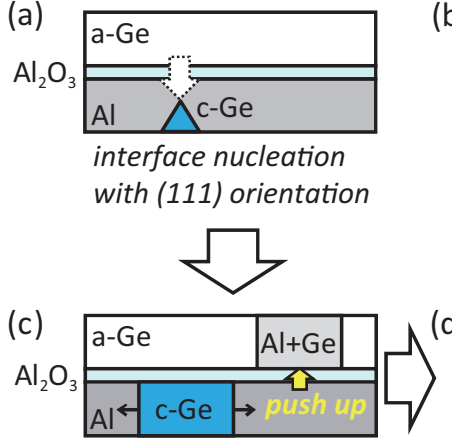

Al-Ge mixed layer formation

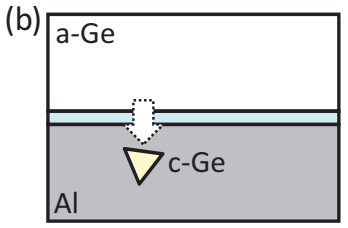

bulk nucleation with random orientation

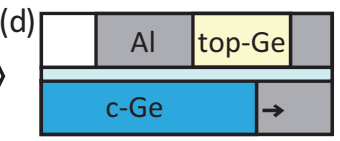

Top-Ge crystallization with Al segregation

Figure 7. Schematic illustrations of the layer exchange process: Ge nucleation in the (a) thin and (b) thick Al layers. (c) Lateral growth of Ge pushing Al up to the a-Ge layer. (d) Formation of the bottom-Ge and top-Ge layers.

in the bulk Al layers as schematically shown in Fig. 7b. This results in the randomly-oriented Ge layers. Therefore, the (111)-orientation fraction decreases with increasing the Ge/Al thickness. For the ALILE process, the number of nuclei per unit area determines the eventual grain size. ${ }^{19,22,23,29}$ The number of nucleation sites increases with Al layer thickness. Therefore, the grain size increases with decreasing the Ge/Al thickness. Such a film-thickness dependence of grain sizes is also observed in solid-phase crystallization of amorphous semiconductor films on insulators. ${ }^{37,38}$

Next, we discuss the formation process of the top-Ge layer. During annealing, Ge atoms diffuse into the $\mathrm{Al}$ layer through the thin $\mathrm{AlO}_{\mathrm{x}}$ membrane. When the Ge concentration in $\mathrm{Al}$ reaches the solubility limit, the Ge nucleation occurs in the Al layer. Continuous supply of $\mathrm{Ge}$ atoms induces the lateral growth of the Ge crystals. This lateral growth stresses the Al layer, and pushes up Al into the a-Ge layer through local perforations of the $\mathrm{AlO}_{\mathrm{x}}$ membrane, as schematically shown in Fig. 7c. ${ }^{22}$ We note that, when the Ge layer is very thin $(30 \mathrm{~nm})$, the total stress to the Al layer is insufficient to perforate the $\mathrm{AlO}_{\mathrm{x}}$ membrane. This results in the stopping of the lateral growth of $\mathrm{Ge}$ as shown in Fig. 5a. The pushed-up Al forms a solid solution with the top a-Ge layer. After that, Ge nuclei occur from the Ge-Al mixed layer, and grow laterally, which results in the segregation of $\mathrm{Al}$, as schematically shown in Fig. 7d. Consequently, the randomly-oriented top-Ge layer is formed on the $\mathrm{AlO}_{\mathrm{x}}$ membrane.
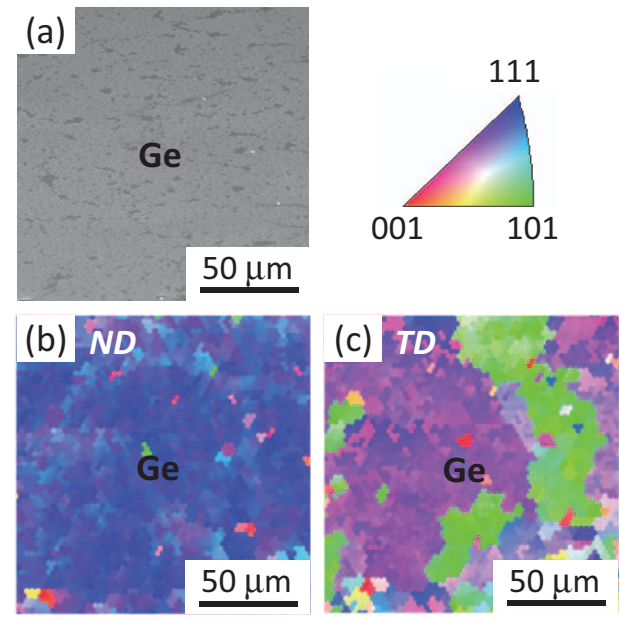

Figure 8. Surface morphologies and crystal orientations for sample $\mathrm{H}$ with 40-nm-thick Ge and 50-nm-thick Al layers. (a) SEM image of the grown $\mathrm{Ge}$ surface. EBSD images obtained along (b) ND and (c) TD to the sample surface. Inserted color-key corresponds to crystal orientations.

Based on the above results, the improvement of the ALILE-Ge layer was investigated. To achieve both of the surface coverage of bottom-Ge layers and the annihilation of top-Ge layers, we employed sample $\mathrm{H}$, prepared with 40-nm-thick Ge and 50-nm-thick Al layers. The results of SEM and EBSD measurements are exhibited in Fig. 8. Fig. 8a indicates that the grown Ge layer covers the almost entire region of the $\mathrm{SiO}_{2}$ surface, while top-Ge layers are present very little. Orientation maps along the ND and TD directions are respectively shown in Figs. 8b and 8c.

Fig. 8b indicates a highly (111)-oriented Ge layer. An EBSD analysis exhibited the (111)-orientation fraction to be $97 \%$. Such a high value is due to the absence of the top-Ge layers. In addition, large grains over tens of microns are suggested by Fig. 8c. The average grain size was calculated to be $70-\mu \mathrm{m}$ diameters. Note that slight color contrasts inside each grain suggest that the orientation gradually changes during the lateral growth of Ge. This phenomenon is probably due to the stress induced at the Ge growth front which pushes Al. Consequently, both of the sufficient Ge coverage on substrates and the annihilation of top-Ge layers were accomplished in this sample, resulting in large-grained Ge layers with highly (111)-orientations.

\section{Conclusions}

We investigated the effects of the Ge/Al thickness on the ALILE process of a-Ge films on insulators. The growth morphology strongly depended on those thicknesses. When the Ge layer is thicker than or equal to the Al layer, randomly oriented top-Ge layers, consisting of random-oriented small grains, were formed on the bottom-Ge layer. In contrast, when the Ge layer is much thinner than the Al layer, the Ge coverage on the substrates becomes insufficient, though the top-Ge layers disappear. The crystal-quality of the bottom-Ge layer was drastically changed by the Ge/Al thickness: The thinner the thickness, the higher the (111)-orientation fraction and the larger the grain size. The lower limit of the thickness was found to be between $30 \mathrm{~nm}$ and $40 \mathrm{~nm}$. This limitation was explained in terms of the Al push up phenomenon during the layer exchange process. Based on these findings, we prepared a sample with 40-nm-thick Ge and 50-nm-thick Al layers. This sample successfully satisfied both of the surface coverage of bottom-Ge layers and the annihilation of top-Ge layers. As a result, the (111)-orientation fraction reached as high as $97 \%$ and the average grain diameter $70 \mu \mathrm{m}$. Therefore, we demonstrated the important role of the Ge/Al thickness in the ALILE process of a-Ge films on insulators. This thickness-controlled ALILE is a promising technique to form high-quality Ge layers on insulators in low-temperature processes.

\section{Acknowledgments}

This work was partially supported by the Murata Science Foundation and by a grant-in-Aid for Scientific Research from the Ministry of Education, Culture, Sport, Science, and Technology in Japan.

\section{References}

1. K. Toko, Y. Ohta, T. Tanaka, T. Sadoh, and M. Miyao, Appl. Phys. Lett., 99, 032103 (2011).

2. C. Y. Tsao, J. W. Weber, P. Campbell, P. I. Widenborg, D. Song, and M. A. Green, Appl. Surf. Sci., 255, 7028 (2009).

3. J. Michel, J. Liu, and C. L. Kimerling, Nature Photonics, 4, 527 (2010).

4. T. Sanada, Y. Nakakita, M. Takenaka, and S. Takagi, J. Apply. Phys., 106, 073716 (2009).

5. T. Nishimura, L. H. Lee, T. Tabata, S. K. Wang, K. Nagashio, K. Kita, and A. Toriumi, Appl. Phys. Express, 4, 064201 (2011).

6. N. Fukata, K. Sato, M. Mitome, Y. Band, T. Sekiguchi, M. Kirkham, J. I. Hong, Z. L. Wang, and R. L. Snyder, ACS Nano, 4, 3807 (2010).

7. E. P. M. Bakkers, J. Dam, S. Franceschi, L. P. Kouwenhoven, M. Kaiser, M. Verheijen, H. Wondergem, and P. Sluis, Nat. Mater., 3, 769 (2004).

8. K. Hamaya, H. Itoh, O. Nakatuka, K. Ueda, K. Yamamoto, M. Itakura, T. Taniyama, T. Ono, and M. Miyao, Phys. Rev. Lett., 102, 137204 (2009).

9. H. Kanno, K. Toko, T. Sadoh, and M. Miyao, Appl. Phys. Lett., 89, 182120 (2006).

10. J. H. Park, M. Tada, P. Kapur, H. Peng, and K. C. Saraswat, J. Appl. Phys., 104, 064501 (2008). 
11. W. Knaepen, S. Gaudet, C. Detavernier, R. L. Meirhaeghe, J. J. Sweet, and C. Lavoie, J. Appl. Phys. 105, 083532 (2009).

12. T. H. Phung, R. Xie, S. Tripathy, M. Yu, and C. Zhu, J. Electrochem. Soc., 157, H208 (2010).

13. T. Sadoh, M. Kurosawa, T. Hagihara, K. Toko, and M. Miyao, ECS. Solid-State Lett., 14, H274 (2012).

14. A. F. Khan, M. Mehmood, A. M. Rana, and T. Muhammad, Appl. Surf. Sci., 256, 2031 (2010).

15. K. Toko, I. Nakao, T. Sadoh, T. Noguchi, and M. Miyao, Solid-State Electron. 53, 1159 (2009).

16. T. Sadoh, H. Kamizuru, A. Kenjo, and M. Miyao, Appl. Phys. Lett. 89, 192114 (2006).

17. O. Nast, T. Puzzer, L. M. Koschier, A. B. Sproul, and S. R. Wenham, Appl. Phys. Lett., 73, 3214 (1998)

18. M. Kurosawa, N. Kawabata, T. Sadoh, and M. Miyao, Appl. Phys. Lett., 95, 132103 (2009).

19. A. Sarikov, J. Schneider, J. Berghold, M. Muske, I. Sieber, S. Gall, and W. Fuhs, J. Appl. Phys. 107, 114318 (2010).

20. M. Jung, A. Okada, T. Saito, T. Suemasu, and N. Usami, Appl. Phys. Express, 3, 095803 (2010).

21. M. Kurosawa, K. Toko, N. Kawabata, T. Sadoh, and M. Miyao, Solid-State Electron., 60, 7 (2011).

22. B. I. Birajdar, T. Antesberger, B. Butz, M. Stutzmann, and E. Spiecker, Scripta Materialia, 66, 550 (2012).

23. A. Okada, K. Toko, K. O. Hara, N. Usami, and T. Suemasu, J. Cryst. Growth, 356 65 (2012).
24. R. Numata, K. Toko, N. Saitoh, N. Yoshizawa, N. Usami, and T. Suemasu, Crystal Growh and Design, 13, 1767 (2013).

25. I. Gordon, L. Carnel, D. V. Gestel, G. Beaucarne, and J. Poortmans, Thin Solid Films, 516, 6984 (2008).

26. B. R. Wu, S. Y. Lo, D. S. Wuu, S. L. Ou, H. Y. Mao, J. H. Wang, and R. H. Horng, Thin Solid Films, 520, 5860 (2012).

27. F. Katsuki, K. Hanafusa, M. Yonemura, T. Koyama, and M. Doi, J. Appl. Phys., 89, 4643 (2001).

28. R. Zanatta and I. Chambouleyron, J. Appl. Phys., 97, 094914 (2005).

29. Z. M. Wang, J. Y. Wang, L. P. H. Jeurgens, F. Phillipp, and E. J. Mittemeijer, Acta. Materialia, 56, 5047 (2008).

30. W. Zhang, F. Ma, T. Zhang, and K. Xu, Thin Solid Films, 520, 708 (2011).

31. S. Hu, A. F. Marshall, and P. C. McIntyre, Appl. Phys. Lett., 97, 082104 (2010).

32. S. Peng, D. Hu, and D. He, Appl. Surf. Sci., 258, 6003 (2012).

33. M. Kurosawa, N. Kawabata, T. Sadoh, and M. Miyao, ECS J. Solid State Sci. and Tech., 1, 144 (2012).

34. K. Toko, M. Kurosawa, N. Saitoh, N. Yoshizawa, N. Usami, M. Miyao, and T. Suemasu, Appl. Phys. Lett., 101, 072106 (2012).

35. K. Toko, N. Fukata, K. Nakazawa, N. Saitoh, N. Yoshizawa, N. Usami, and T. Suemasu, J. Cryst. Growth, 372, 189 (2013).

36. A. A. Stekolnikov, J. Furthmuller, and F. Bechstedt, Phys. Pev. B, 65, 115318 (2002).

37. P. Germain, K. Zellama, S. Squelard, J. C. Bourgoin, and A. Gheorghiu, J. Appl. Phys., 50, 6986 (1979).

38. C. Spinella, S. Lombardo, and F. Priolo, J. Appl. Phys., 84, 5383 (1998). 